\title{
Probing structure in colloidal gels of thermoreversible rodlike virus particles: Rheology and scattering
}

\author{
Naveen Krishna Reddy and Zhenkun Zhanga) \\ Department of Chemical Engineering, KU Leuven, \\ University of Leuven, W. de Croylaan 46, B-3001 Leuven, Belgium \\ M. Paul Lettinga and Jan K. G. Dhont \\ Institute for Complex Systems-3, Forschungszentrum Jülich, \\ D-52425 Jülich, Germany \\ Jan Vermant ${ }^{\text {b) }}$ \\ Department of Chemical Engineering, KU Leuven, \\ University of Leuven, W. de Croylaan 46, \\ B-3001 Leuven, Belgium
}

(Received 27 October 2011; final revision received 21 May 2012; published 19 June 2012)

\begin{abstract}
Synopsis
Aggregated suspensions of rodlike particles are commonly encountered in soft biological materials and their solidlike response at extremely low volume fractions is also exploited technologically. Understanding the link between the physicochemical parameters such as size, aspect ratio, volume fraction, and interparticle forces with the resulting microstructure and the subsequent rheological response remains challenging. In the present work, suspensions of monodisperse rodlike virus particles, whose surface is modified by grafting with a thermoreversible polymer poly( $N$-isopropylacrylamide), are used as a model system. The repulsive and attractive contributions to the total interaction potential can be changed independently by varying the ionic strength and the temperature. The effects of these changes on the strength and structure of gels have been studied near the gel transition using a combination of rheological and scattering measurements. Rheological measurements of the near critical gel properties as a function of concentration and ionic strength proved to be more sensitive compared to scattering in resolving the structural differences. A percolating structure can be formed at very low volume fractions, which show a weak dependence on the ionic strength with the anisotropy of the repulsive interactions playing the main role in creating more "open" structures. The intrinsic stiffness of the rodlike particles does not affect the moduli of the gel states very strongly. C 2012 The Society of Rheology. [http://dx.doi.org/10.1122/1.4728335]
\end{abstract}

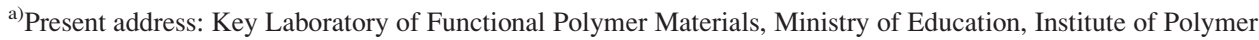
Chemistry, Nankai University, Tianjin 300071, P. R. China.

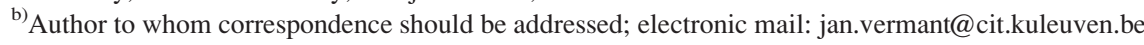




\section{INTRODUCTION}

Anisotropic rodlike particles can be used as basic building blocks for efficiently building up strong materials at low volume fractions. Examples can be found in material classes ranging from the cytoskeleton in cellular materials [Elson (1988); Lin et al. (2007)] to rheology control agents in coatings [Kheirandish et al. (2008)] and structured consumer products [Maynard (2006)]. Historically, rodlike and disklike clay particles were used in pottery to induce plasticity while rendering the pottery permeable to air [Kingery et al. (1976)]. More recently, carbon nanotubes have been introduced to strengthen polymer composites [Srivastava et al. (2003)], and rodlike polymers have been designed to create super adsorbing, high water content hydrogels [Schultz et al. (2009)]. The most prominent feature of rodlike particles in creating strong structures is their anisotropic excluded volume which lowers the percolation threshold [Schilling et al. (2007); Otten and van der Schoot (2009); Mohraz et al. (2004); Basavaraj et al. (2009)]. In the presence of an attractive interaction the percolation threshold can be lowered even further, hence possibly rendering sticky rods as ideal building blocks for self-assembly of gel-like materials at low solid content or volume fraction [Schilling et al. (2007)].

Apart from volume fraction, the properties of the gel can be controlled by the aspect ratio, the interaction potential and the inherent flexibility of the constituent particles. A wide range of attractive rodlike systems have been studied, from bentonite gels [Alderman et al. (1991)] over boehmite gels [Wierenga et al. (1998); van Bruggen et al. (2002); ten Brinke et al. (2007)] to actin gels [Wachsstock et al. (1994); Tempel et al. (1996); Tharmann et al. (2007)] and more recently the industrially relevant polyamide gels [Wilkins et al. (2009)]. Solomon and Spicer critically reviewed the topic identifying the generic features of rodlike gel systems [Solomon and Spicer (2010)]. Even though some general trends are emerging, the progress in understanding the self-assembly of these rods is being hampered by the lack of suitable model systems, monodisperse in size and aspect ratio with known flexibility and tunable interaction potentials.

In the present work, monodisperse rodlike particles grafted with a thermosensitive polymer poly( $N$-isopropylacrylamide) (PNIPAM) are used as a model system [Zhang et al. (2009); Huang et al. (2009)]. Two types of rodlike particles, wild type $f d$-virus and its $f d-Y 21 M$ mutant are studied. These are model rodlike particle systems in equilibrium [Dogic and Fraden (2000)] and in shear flow [Lettinga et al. (2005); Graf et al. (1993)]. The geometric properties are same for both the viruses, but their flexibility is different with $f d$-virus being semiflexible and the $f d-Y 21 M$ being stiffer due to a slight difference in protein structure [Barry et al. (2009)]. The overall interaction potential in these model systems stems from a balance between the electrostatic repulsions, caused by surface charges on the rods, and the properties of the temperature sensitive grafted polymeric layer. These interactions can be tailored. The strength of the electrostatic repulsion depends on $p \mathrm{H}$ and ionic strength. Due to the presence of both carboxyl and amine groups, the surface charge of the $f d$-virus can be varied from positive to negative. At the physiological $p \mathrm{H}$ of 8.2 the virus carries a negative surface charge density of about $10 \mathrm{e} /$ $\mathrm{nm}$. The thickness of the electrostatic double layer around the particles can hence be varied with the ionic strength [Grelet and Fraden (2003)]. The resulting electrostatic interaction potential is spatially anisotropic. For rigid rods, the dependence on relative orientation has been predicted, and depends on the relative orientation of the particles, with parallel and perpendicular orientations having strong and weak repulsions, respectively [Sparnaay (1959); Stroobants et al. (1986)].

The thermoreversible polymer PNIPAM has both hydrophilic and hydrophobic parts. The hydrophilic parts find their origin from interaction of anions in the solvent with 
oxygen and nitrogen rich parts of the PNIPAM. The local hydrophobicity comes from the isopropyl groups and the polymethylene backbone [Pelton (2010)]. A sharp change from a coil to a globular conformation occurs as the temperature is increased above a critical temperature [Schild (1992); Pelton (2010)]. For a PNIPAM polymer solution, the critical temperature is referred to as the volume phase change temperature $\left(\mathrm{T}_{\mathrm{VPT}}\right)$ [Tanaka et al. (1980)]. For PNIPAM grafted onto surfaces at low temperatures the coil state of PNIPAM gives rise to a steric barrier yielding a sterically stabilized suspension, whereas at higher temperatures the collapsed PNIPAM chains expose more of the hydrophobic regions to the solvent which induces a strong short ranged attraction. The model system of PNIPAM grafted $f d$-virus particles in aqueous media can therefore be thermoreversibly switched between a suspension and a colloidal gel [Zhang et al. (2009); Huang et al. (2009)]. The attractive interaction potential can be expected to be at a maximum when the rods are parallel and at a minimum when they are perpendicular to each other. As the relative balance between the electrostatic and polymeric interactions can be tuned by ionic strength and temperature, the effects of varying the total interaction potential on the self-assembly in the gel state can be investigated. Apart from the strength of the interaction, the spatial dependence of the potential will also change. For systems in which the electrostatic interaction is "switched on" a more open structure can be expected, compared to the case when only a steric repulsion exists prior to the rapid gelation. These effects can be expected to be less pronounced for particles that are flexible.

Most studies on aggregated suspensions of rodlike particles have focused either on determination of the structure at low volume fractions by studying the fractal dimension and the structural growth mechanisms using light scattering [Wierenga et al. (1998); Mohraz et al. (2004); Hobbie et al. (2009); Lin et al. (2007)] or on the rheological properties of fully developed gels, i.e., systems deep in the gel regime [Solomon and Spicer (2010)]. In the present work, the effect of changing the interactions is studied by measuring the rheological and structural properties near the gel transition regime. For the $f d$ PNIPAM suspensions at the gel point, one obtains what Winter and Chambon (1986) called "critical gels" [Winter and Chambon (1986)]. They are characterized by a selfsimilar relaxation modulus,

$$
G(t)=S t^{-n},
$$

where $G(t)$ is the relaxation modulus, $S$ and $n$ are the two fitting parameters that characterize the gel [Chambon and Winter (1987); Winter (2002)]. The parameter $S$ represents the strength of the gel and power-law exponent $n$ provides the structural information. This power law implies that relaxation processes appear to be the same on all length scale. Consequently, the time and frequency dependence of the rheological properties are simple power laws. For polymeric systems, studies in the critical gel regime have provided insight into the nature of the gelation [Winter and Chambon (1986); Chambon and Winter (1987); Venkataraman and Winter (1990); Ng and Mckinley (2008)] and the gel structure. For suspensions, not all gels go through a critical gel state [Verduin et al. (1996); Mohraz and Solomon (2006); Yin and Solomon (2008)], but we will demonstrate that the $f d$-PNIPAM suspensions exhibit a power-law type relaxation spectrum over the entire range of experimentally accessible time scales. For colloidal gels, the power law reflects the presence of self-similar colloidal clusters over a broad range of length scales. In order to study the properties of the developed gel with sufficient experimental accuracy, it was necessary to surpass the gel point by some extent. In this regime, the magnitude of the linear viscoelastic storage moduli and its dependence on the frequency will be compared to the results obtained from scattering experiments. 


\section{MATERIALS AND METHODS}

\section{A. System}

The $f d$-virus and its $f d$ - $Y 21 M$ mutant are filamentous bacteriophages made of single stranded circular deoxyribose nucleic acid (DNA) covered with 2700 coat proteins. The virus particles are monodisperse, with a contour length of $880 \mathrm{~nm}$, a bare rod diameter $D_{\text {bare }}=6.6 \mathrm{~nm}$, and a molecular weight of $1.64 \times 10^{7} \mathrm{~g} / \mathrm{mol}$. The persistence length of $f d$-virus and $f d-Y 21 M$ are 2800 and $10000 \mathrm{~nm}$, respectively [Barry et al. (2009)]. Both virus particles are negatively charged $(10 \mathrm{e} / \mathrm{nm})$ at $8.2 \mathrm{pH}$ and form a stable suspension in an aqueous buffer at all ionic strengths due to the electrostatic repulsion. Batches of both the viruses were grown and purified using a standard biological protocol [Sambrook and Russell (2001)]. The possibility for inducing an attractive interaction is realized by grafting a thermoreversible polymer (PNIPAM) on the viruses via their coat proteins, resulting in a hybrid virus-PNIPAM particle. The molecular weights of PNIPAM used are 7 and $10 \mathrm{kDa}$ and the grafting density of PNIPAM is around 400 PNIPAM chains per virus particle [Zhang et al. (2009)]. The detailed procedure for grafting PNIPAM onto the virus has been reported earlier [Zhang et al. (2009)]. The PNIPAM-grafted virus suspensions were extensively dialyzed against, respectively, 1 and $5 \mathrm{mM}$ Tris buffer and a $105 \mathrm{mM}$ Tris- $\mathrm{NaCl}$ buffer at a $p \mathrm{H}$ of 8.2. The concentrations of $f d$-PNIPAM suspensions were determined using absorption spectroscopy with an optical density (OD) for a $1 \mathrm{mg} / \mathrm{ml}$ virus solution of $\mathrm{OD}_{10 \mathrm{~mm}}^{269 \mathrm{~nm}}=3.84$ and 3.63 for of $f d$-virus and $f d-Y 21 M$, respectively [Barry et al. (2009)].

When estimating the interaction potential between the particles we start from the electrostatic repulsion of the bare $f d$-virus with a net linear charge density $e^{-1} / \AA$, which can be tuned by screening the effect of surface charge by the addition of salt. This repulsive part of the interaction potential for center-to-center distance $\left(\mathrm{X}_{\text {core-core }}\right)$ for two parallel rods is plotted in Fig. 1(a) for the three ionic strengths used here [Tang and Fraden (1995)]. The effect of the electrostatic repulsion can be used to rescale the particle diameter to an effective hard core diameter $D_{\text {eff }}^{\mathrm{ES}}[$ Tang and Fraden (1995)]. Ionic strengths of 1, 5 , and $105 \mathrm{mM}$ result in effective radii of $D_{\text {eff }}^{\mathrm{ES}}=11,30$, and $63 \mathrm{~nm}$, respectively. For the PNIPAM coated particles, the electric field strength may be altered by a change in the local dielectric constant in the polymer brush, reducing the effective diameter somewhat.

(a)

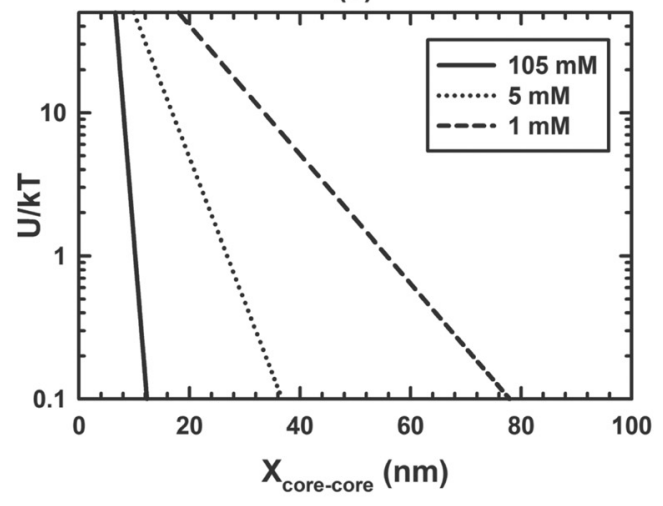

(b)

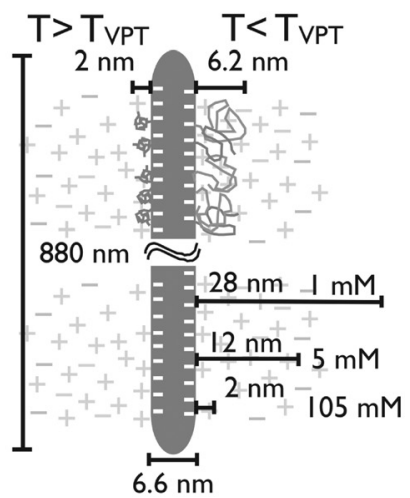

FIG. 1. (a) Electrostatic potential $(\mathrm{U} / \mathrm{kT})$ as a function of the center-to-center distance $\left(\mathrm{X}_{\text {core-core }}\right)$ for two uncoated $f d$-viruses oriented perpendicular to each other for the different ionic strengths [Tang and Fraden (1995)]. (b) Cartoon of $f d$-virus with the relevant dimensions for the effective electrostatic double layer thickness and the thickness of PNIPAM layer below and above the volume phase change temperature. 
The relevant dimensions of $f d$-virus, PNIPAM polymer layer thickness (below and above $\mathrm{T}_{\mathrm{VPT}}$ ), and the electrostatic double layer thickness in different ionic strengths are shown in Fig. 1(b).

Below the VPT $\left(T<T_{\mathrm{VPT}}\right)$, the grafted PNIPAM causes an additional steric repulsion, with $D_{\text {eff }}^{\text {Ster. }}=D_{\text {bare }}+4 R_{g}$, where $R_{g}$ is the radius of gyration. At low ionic strength the electrostatic contribution still dominates $\left(D_{\text {eff }}^{\text {Ster. }}<D_{\text {eff }}^{\mathrm{ES}}\right)$. Above a critical value of the ionic strength the steric repulsion becomes the most prominent $\left(D_{\text {eff }}^{\text {Ster. }}>D_{\text {eff }}^{\mathrm{ES}}\right)$. In this case a further increase of the ionic strength does not change the effective diameter. As a result, the isotropic to nematic transition, which depends on the effective diameter [Onsager (1949)], will become independent of the ionic strength exactly at the ionic strength where $D_{\text {eff }}^{\text {Ster. }}=D_{\text {eff }}^{\mathrm{ES}}$. Huang et al. (2009) found $D_{\text {eff }}^{\text {Ster. }} \sim 17 \mathrm{~nm}$ for $f d$-PNIPAM with $10 \mathrm{kDa}$ PNIPAM at room temperature, giving a steric repulsive layer of about $5 \mathrm{~nm}$. It can be inferred from Fig. 1(b) that only for the sample at an ionic strength $105 \mathrm{mM}$ the interactions are certainly dominated by steric repulsion.

The polymer contribution to the interaction between two neighboring PNIPAMgrafted rods becomes attractive when the temperature is increased above the VPT. There is, to our knowledge, no generally accepted model to describe this short ranged interaction quantitatively. The range of the attractive potential is set by the density distribution of the polymer, and is therefore linked to the polydispersity and chain confirmation of the collapsed PNIPAM. As can be inferred from Fig. 1, for samples with the ionic strength of 5 and $1 \mathrm{mM}$ the collapsed PNIPAM layer should be buried in the repulsive electrostatic double layer. We will show, however, that even for these low ionic strengths systems, gelation will occur, although $T_{\mathrm{VPT}}$ does shift to a higher temperature. Note that so far only the interaction between rods with a perpendicular orientation was considered. The repulsive interaction is expected to become stronger when rods rotate towards a parallel orientation, while the overall attractive interactions also increase as well. We conjecture that for the lowest ionic strength the spatial anisotropy of the electrostatic interaction may lead to a decreased probability for parallel orientations which may be locked in during gelation and result into more "open" structures at low ionic strength. It is, however, not clear how important effects of flexibility will be. The only piece of experimental evidence is that the isotropic-nematic phase transition for $f d-Y 21 M$ virus occurs at slightly lower concentrations compared to the bare $f d$-virus particles due to the higher rod stiffness [Barry et al. (2009)].

The different suspension compositions studied in this work, both in terms of particle concentration and ionic strengths, are tabulated in Table I. For dynamic light scattering (DLS), experiments were done at much lower concentrations, starting around the overlap concentration, which is $0.07 \mathrm{mg} / \mathrm{ml}$ for $f d$-PNIPAM particles. The concentrations are listed both in $\mathrm{mg} / \mathrm{ml}$ as well as the volume fractions for the sake of comparison.

\section{B. Rheometry}

Rheological experiments were performed using both strain and stress controlled rheometers. Stress relaxation experiments were carried out on an ARES strain controlled rheometer equipped with a $100 \mathrm{~g} / \mathrm{g} \mathrm{cm}$ transducer (Advanced Rheological Expansion System, TA instruments, USA). All remaining rheological measurements were performed using an MCR 501 stress controlled rheometer (Paar Physica, Austria). Rough and smooth cone geometries with a diameter of $25 \mathrm{~mm}$ and cone angles of 0.07 and $0.04 \mathrm{rad}$ were used. Measurements using rough and smooth plates with different cone angles gave identical results, confirming the absence of slip [Yoshimura and Prud'homme (1988); Buscall et al. $(1993,2010)]$. The temperature was varied from 25 to $40^{\circ} \mathrm{C}$ at a heating 
TABLE I. Properties of the suspensions studied: ionic strength $I$, effective diameter $D_{\text {eff }}(\mathrm{nm})$, concentration $C$ $(\mathrm{mg} / \mathrm{ml})$, and effective volume fraction $\Phi_{\text {eff }}$.

\begin{tabular}{|c|c|c|c|c|c|c|}
\hline$I$ & \multicolumn{2}{|c|}{$1 \mathrm{mM}$} & \multicolumn{2}{|c|}{$5 \mathrm{mM}$} & \multicolumn{2}{|c|}{$105 \mathrm{mM}$} \\
\hline \multirow[t]{7}{*}{$D_{\text {eff }}$} & \multicolumn{2}{|c|}{$25.8 \mathrm{~nm}$} & \multicolumn{2}{|c|}{$15.6 \mathrm{~nm}$} & \multicolumn{2}{|c|}{$10.5 \mathrm{~nm}$} \\
\hline & $\mathrm{C}(\mathrm{mg} / \mathrm{ml})$ & $\Phi_{\text {eff }}$ & $\mathrm{C}(\mathrm{mg} / \mathrm{ml})$ & $\Phi_{\text {eff }}$ & $\mathrm{C}(\mathrm{mg} / \mathrm{ml})$ & $\Phi_{\text {eff }}$ \\
\hline & 1.61 & $8.7 \times 10-3$ & 2.0 & $3.8 \times 10-3$ & 1.61 & $1.3 \times 10-3$ \\
\hline & 2.56 & $13.8 \times 10-3$ & 3.0 & $5.8 \times 10-3$ & 2.64 & $2.1 \times 10-3$ \\
\hline & 3.55 & $19.2 \times 10-3$ & 4.0 & $7.7 \times 10-3$ & 3.61 & $2.9 \times 10-3$ \\
\hline & \multirow[t]{2}{*}{4.57} & $24.7 \times 10-3$ & & & 4.44 & $3.6 \times 10-3$ \\
\hline & & & & & 7.74 & $6.3 \times 10-3$ \\
\hline
\end{tabular}

rate of $25^{\circ} \mathrm{C} / \mathrm{min}$ using a Peltier element to control temperature. The temperature of the sample in the gap was measured using an externally calibrated thermocouple (hypodermic needle, type T Copper-Constantan, Omega, Stamford CT). All measurements were performed using a double lock solvent trap to reduce evaporation [Sato and Breedveld (2005)]. The protocol used in all rheological measurements is as follows: the exact volume required to properly fill the geometry of $f d$-PNIPAM suspension was loaded on the Peltier at $25^{\circ} \mathrm{C}$. The upper geometry was lowered while rotating at a constant speed of 10 $\mathrm{rev} / \mathrm{min}$ to uniformly spread the sample underneath. Once the upper geometry came to rest, the temperature of the sample was increased to the measuring value. After the set temperature was reached, the sample was allowed to equilibrate for $3 \mathrm{~min}$ at the set temperature before starting the measurements. Most rheological experiments were performed at $\Delta T=T-T_{\mathrm{VPT}}=4^{\circ} \mathrm{C}$ to obtain a sufficient torque that was above the transducer limit but still being in the critical gel regime, as will be discussed later.

\section{Scattering}

DLS and static light scattering (SLS) measurements were performed on an ALV CGS3 compact goniometer system equipped with a multitau digital correlator (ALV/LSE5003, Langen, Germany). The light source was a $10 \mathrm{~mW}$ He-Ne laser of wavelength $\lambda=632.8 \mathrm{~nm}$. For the DLS and SLS measurements, $0.6 \mathrm{ml}$ of $f d$-PNIPAM solution was loaded into a $5 \mathrm{~mm}$ diameter glass tube that was flame sealed to prevent evaporation. The glass tubes were placed inside the setup $8 \mathrm{~h}$ before the measurements to ensure that all the dust particles in the index matching vat had settled. The temperature was controlled using a fluids bath to within $\pm 0.1^{\circ} \mathrm{C}$. Samples were equilibrated at the set temperature for $30 \mathrm{~min}$ prior to any measurement. Measurements were carried out at a scattering angle of $90^{\circ}$ in the case of DLS and from 30 to $120^{\circ}\left(6.835 \times 10^{-3} \mathrm{~nm}^{-1}<q<2.28 \times 10^{-2}\right.$ $\mathrm{nm}^{-1}$ ) in the case of SLS. $q$ is the scattering vector given by $q=4 n_{i} \pi \sin (\theta / 2) / \lambda$, with $n_{i}$ the refractive index of the solution and $\theta$ the scattering angle. To access smaller $q$ values $\left(2.27 \times 10^{-4} \mathrm{~nm}^{-1}<q<3.4 \times 10^{-3} \mathrm{~nm}^{-1}\right)$, a home built small angle light scattering (SALS) setup in combination with an MCR 300 stress controlled rheometer with parallel plate geometry was used [Hoekstra et al. (2005)]. In this setup, scattering from the sample was captured and directly guided on to a CCD chip using a set of lenses to obtain a sufficient resolution. The bottom and the top glass plates were made of quartz coated with indium tin oxide (ITO) to promote thermal conduction. A $5^{\circ} \mathrm{C}$ temperature gradient from the outer diameter of the glass plate to the center of the measuring device was, however, still measured. Light scattering data were collected when the temperature at the beam spot, measured by an external thermocouple (hypodermic needle) on the opposite 
side of the measuring device reached the set temperature (to avoid damaging the gel at the beam spot). To access larger values of $q$ compared to the SLS $\left(4.5 \times 10^{-2}\right.$ $\mathrm{nm}^{-1}<q<1.13 \mathrm{~nm}^{-1}$ ), small angle $\mathrm{x}$-ray scattering (SAXS) measurements were performed at high brilliance beam-line (ID02) at the European Synchrotron Radiation Facility in Grenoble, France. Here, the sample was loaded in a Couette cell equipped with a solvent trap and the temperature was controlled using a fluids bath [Panine et al. (2002)].

\section{EXPERIMENTAL RESULTS AND DISCUSSION}

\section{A. Determination of the gel temperature}

The gelation temperature $\left(T_{\mathrm{VPT}}\right)$ for $f d$-PNIPAM at different ionic strengths was determined by DLS and rheology. In DLS, $T_{\mathrm{VPT}}$ was defined as the temperature at which the suspension showed a transition from ergodic to nonergodic state [Mohraz and Solomon (2006)]. For the rheological measurements, $T_{\mathrm{VPT}}$ was taken as the temperature at which $\tan \delta=\left(G^{\prime \prime} / G^{\prime}\right)$ became independent of the applied frequency $(\omega)$ [Winter and Chambon (1986)].

\section{DLS}

At low temperatures $\left(T<T_{\mathrm{VPT}}\right), f d$-PNIPAM suspensions are ergodic, a state in which the time averaged intensity correlation function $\left(g_{2}(t, q)-1\right)$ is equal to the ensemble averaged intensity correlation function. At time $\mathrm{t}=0, g_{2}(0)-1$ for an ergodic system is close to 1 . For $T>T_{\mathrm{VPT}}$ the $f d$-PNIPAM suspension transforms into a gel where the movements of the particles get constrained and the system becomes nonergodic. At time $\mathrm{t}=0, g_{2}(0)-1$ for a nonergodic system typically drops below one as the system becomes spatially heterogeneous [Matsunaga and Shibayama (2007)]. The gelation temperature can hence be determined from the initial value of $g_{2}(0)-1$. Figure 2(a) shows $g_{2}(0)-1$ as a function of temperature for $f d$-PNIPAM in buffers of $I=1$ and $105 \mathrm{mM}$ ionic strength. The gel temperatures for $f d$-PNIPAM in 1,5 , and $105 \mathrm{mM}$ buffers were found to be 35,34 , and $32^{\circ} \mathrm{C}$, respectively, as shown in Fig. 2(a) (inset). DLS was also used to determine the minimum concentration of $f d$-PNIPAM particles needed for

(a)

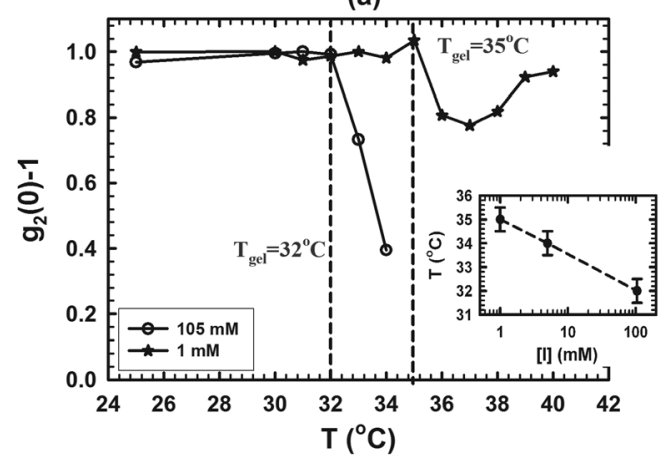

(b)

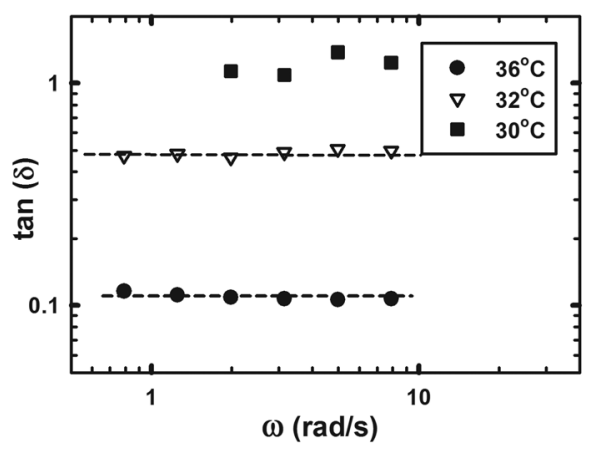

FIG. 2. Gel point determination. (a) The initial value of the time averaged intensity auto correlation function at $\mathrm{t}=0\left(g_{2}(0)-1\right)$ as a function of temperature for 3.0 and $3.61 \mathrm{mg} / \mathrm{ml} f d$-PNIPAM in 1 and $105 \mathrm{mM}$ ionic strength buffers, respectively. (b) $\tan \delta(\omega)$ as a function of frequency at different temperatures for $3.61 \mathrm{mg} / \mathrm{ml}$ $f d$-PNIPAM in $105 \mathrm{mM}$ ionic strength. The dashed lines indicate the independence of $\tan \delta(\omega)$ with respect to frequency at the gelation temperature. Inset in (a) shows $T_{\mathrm{VPT}}$ for $f d$-PNIPAM system as a function of ionic strength. 
gelation. The DLS experiments revealed that the gelation occurs only if the $f d$-PNIPAM concentration exceeds the overlap concentration $C^{*} \approx 0.07 \mathrm{mg} / \mathrm{ml}$. The overlap concentration $\mathrm{C}^{*} \approx 0.07 \mathrm{mg} / \mathrm{ml}$ is equal to $\Phi_{\text {eff }}^{*} \approx 5.6 \times 10^{-5}$ for $f d$-PNIPAM in $105 \mathrm{mM}$, which makes this one of the lowest volume fraction gels ever achieved [Solomon and Spicer (2010)]. The gelation for concentrations above $C^{*}$ also suggests that the $f d$-PNIPAM gels form a homogeneous gel. Similar homogeneous gels have been seen for cross-linked actin networks [Tharmann et al. (2007)].

\section{Rheology}

For near critical gels under small amplitude oscillatory shear, both the storage and loss modulus display the same power-law behavior with respect to frequency,

$$
G^{\prime}(\omega) \propto G^{\prime \prime}(\omega) \propto \omega^{n}
$$

where the exponent $n$ is related to the structure of the gels and varies between 0 and 1 . This results in $\tan \delta(\omega)$ being independent of frequency, a criterium that can be used to determine the gel temperature [Chambon and Winter (1987)],

$$
\tan \delta(\omega)=\frac{G^{\prime \prime}(\omega)}{G^{\prime}(\omega)}=\tan \left(\frac{n \pi}{2}\right) .
$$

Figure 2(b) shows $\tan \delta$ as a function of frequency at different temperatures for $3.61 \mathrm{mg} /$ $\mathrm{ml} f d$-PNIPAM in a $105 \mathrm{mM}$ ionic strength buffer. The gelation temperature is identified as $32^{\circ} \mathrm{C}$, which is identical to the result obtained from the DLS experiments. $\mathrm{T}_{\text {gel }}$ for the $f d$-PNIPAM suspensions at an ionic strength of 1 and $5 \mathrm{mM}$ are 35 and $34^{\circ} \mathrm{C}$, respectively, again in excellent agreement with the results of DLS. As can be seen in Fig. 2(b), the critical gel regime extends slightly beyond the gelation temperature, at least up to $\Delta T=4^{\circ} \mathrm{C}$. To increase the signal to noise ratio during the rheological measurements, the experiments were performed at $\Delta T=4^{\circ} \mathrm{C}$, unless stated otherwise.

\section{B. Determination of gel structure by scattering}

The discussion on the interaction potential gave an idea about how the interactions at the particle level can be tailored. This should be reflected in the microstructure. To probe the structure at the network level, scattering experiments were performed on suspensions and gels in buffers of $I=1$ and $105 \mathrm{mM}$. Figure 3(a) shows the static scattered intensity as a function of the normalized scattering vector $(q L)$ for $3.5 \mathrm{mg} / \mathrm{ml} f d$-PNIPAM suspension and gel at ionic strength of $105 \mathrm{mM}$ and a $1 \mathrm{mM}$ ionic at $T=25^{\circ} \mathrm{C}$. The SAXS data for $f d$-PNIPAM suspensions show a $q^{-4}$ and $q^{-1}$ dependency at large and small $q L$, respectively, reflecting the form factor of the randomly orientated rods [Solomon and Spicer (2010)]. For stable suspensions at even smaller $q L$ values, $q L<30$ a $q^{-2.5}$ dependency is observed experimentally. The $q^{-2.5}$ dependency at small $q L$ is caused by the liquid like structure factor as the concentration of $f d$-PNIPAM particles is about 100 times the overlap concentration. Similar liquidlike structures have been observed for suspensions of bare $f d$-virus particles at low ionic strengths [Hagenböchle et al. (1990); Schulz et al. (1991)].

As the temperature is increased above $T_{\mathrm{VPT}}\left(\Delta T=4^{\circ} \mathrm{C}\right)$ the scattering from the gel at large $q L$ (SAXS) still displays a $q^{-4}$ dependency. For smaller values of $q L$ an exponent of -1.7 is observed, which is caused by the presence of aggregates that have a higher scattering power compared to individual $f d$-PNIPAM particles. For $q L<30$, the 

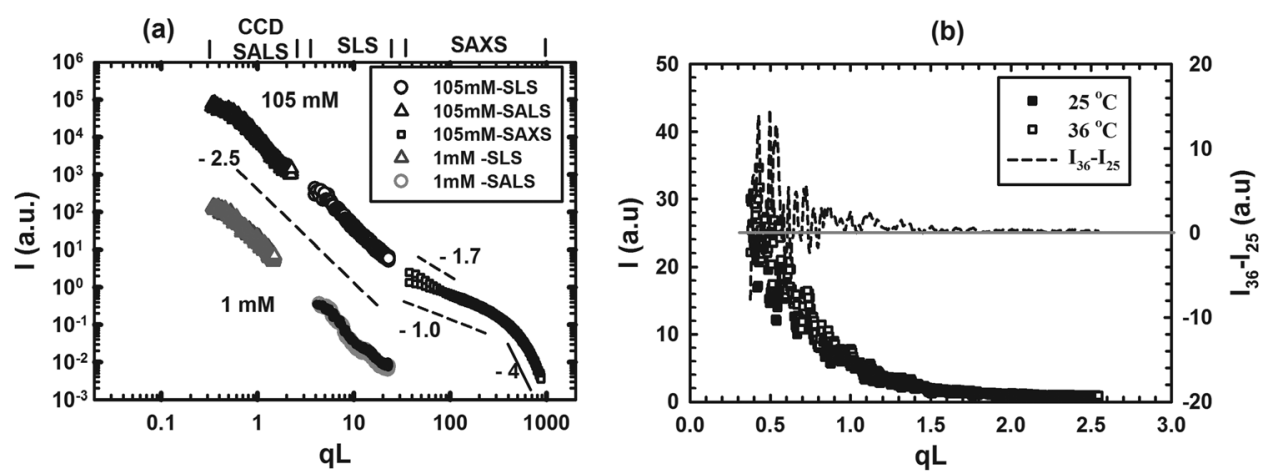

FIG. 3. (a) Combined small angle light and $\mathrm{x}$-ray scattering data for $3.5 \mathrm{mg} / \mathrm{ml} f d$-PNIPAM suspensions in buffers at $I=1$ and $105 \mathrm{mM}$ ionic strength at different temperatures (curves off-set for the sake of clarity). (b) Difference in the scattered light intensity between $f d$-PNIPAM suspensions above and below the VPT in $I=105 \mathrm{mM}$.

difference in scattering intensity between $f d$-PNIPAM suspension and the gel is extremely small. The small scattering difference suggests that, as the temperature is increased above $T_{\mathrm{VPT}}$, the sticky $f d$-PNIPAM particles in suspension gets locked into a homogeneous network, the dynamic arrest being consistent with the interaction potential calculations. The gelled $f d$-PNIPAM suspension shows the same $q^{-2.5}$ dependence at low $q$. A careful examination of scattering intensity from $f d$-PNIPAM suspension and the gel, as presented in Fig. 3(b), shows a small difference (dashed line) in $q L<3.0$ range. The light scattered from the gel is slightly higher than that of the stable suspension. The scattering data from gels in $I=1$ and $105 \mathrm{mM}$ both show a $q^{-2.5}$ dependence, Fig. 3(a). This implies that both the gels have a similar structure.

\section{Rheological characterization in the linear viscoelastic regime}

The $f d$-PNIPAM gels are first characterized in the linear viscoelastic regime, which have been determined using oscillatory strain sweep experiments. The results obtained in the critical gel regime (close to the gel point at $\Delta T=T-T_{\mathrm{VPT}}=4^{\circ} \mathrm{C}$ ) are analyzed using the approach by Winter and Chambon for near critical gels [Chambon and Winter (1987)]. It is based on a power-law model with two parameters, the gel strength $(S)$ and the power-law exponent $(n)$.

\section{Small amplitude oscillatory shear experiments}

a. Strain sweep. The linear viscoelastic limit is determined by performing strain sweep experiments at $\Delta T=4^{\circ} \mathrm{C}$. The strain amplitude is varied from $1 \times 10^{-3}$ to 10 at a constant frequency $(\omega)$ of $6.28 \mathrm{rad} / \mathrm{s}$. Figure 4 shows $G^{\prime}$ and $G^{\prime \prime}$ as a function of strain amplitude for $4.57 \mathrm{mg} / \mathrm{ml}$ and $4.44 \mathrm{mg} / \mathrm{ml}$ of $f d$-PNIPAM gels in buffers with $I=1$ and $105 \mathrm{mM}$ ionic strength, respectively. In the linear limit $f d$-PNIPAM gel in $I=105 \mathrm{mM}$ shows higher moduli as compared to gel in $I=1 \mathrm{mM}$ in agreement with the weaker electrostatic repulsion. The linear to nonlinear viscoelastic transition occurs between a strain amplitude of $0.1-1$ for both ionic strengths. The increase in $G^{\prime \prime}$ in the nonlinear regime above its average value close to the crossover is typical for the yielding of a particulate gel [Buscall et al. (1998)].

b. Frequency sweep. The effect of temperature on the strength and structure of the gels is determined by performing frequency sweep experiments at a constant strain 


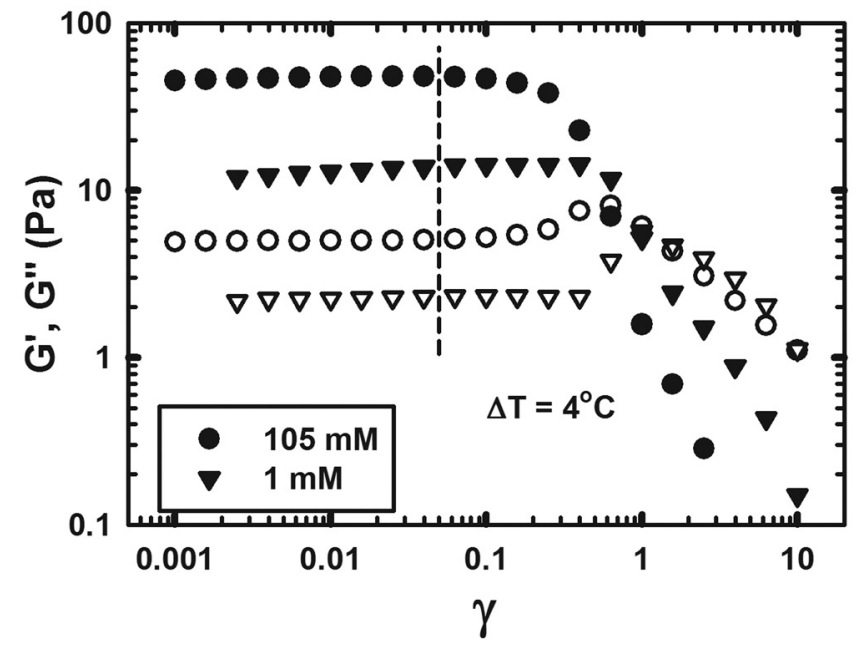

FIG. 4. Storage modulus $\left(\mathrm{G}^{\prime}\right.$, closed symbols) and loss modulus $\left(\mathrm{G}^{\prime \prime}\right.$, open symbols) plotted as a function of strain amplitude $(\gamma)$ for 4.57 and $4.44 \mathrm{mg} / \mathrm{ml}$ of $f d$-PNIPAM in $I=1$ and $105 \mathrm{mM}$ buffers, respectively $\left(\omega=6.28 \mathrm{rad} / \mathrm{s}, \Delta T=4^{\circ} \mathrm{C}\right)$

amplitude of 0.05 . Figure 5(a) shows the storage modulus as a function of frequency for $3.61 \mathrm{mg} / \mathrm{ml}$ of $f d$-PNIPAM in $I=105 \mathrm{mM}$ at different temperatures. The lines in Fig. 5(a) are fits to the transform of Eq. (1) in the frequency domain, given by [Venkataraman and Winter (1990)],

$$
G^{\prime}(\omega)=\Gamma(n-1) \cos \frac{n \pi}{2} S \omega^{n} .
$$

Figure 5(b) shows the evolution of the power-law exponent obtained from Fig. 5(a) as a function of temperature. Increasing the temperature by $1{ }^{\circ} \mathrm{C}$ from the critical point changes slope $n$ sharply. Every further increase in temperature only increases the magnitude of the storage modulus without altering $n$. The power-law exponent $n$ changes from 0.35 at the critical gel point $\left(\Delta T=0^{\circ} \mathrm{C}\right)$ to 0.2 at $\Delta T=1{ }^{\circ} \mathrm{C}$ and decreases very slowly as the temperature is further increased. A sharp change in $n$ at the critical gel point

(a)

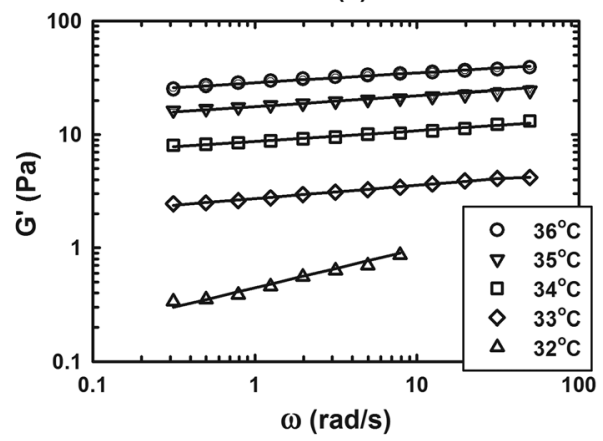

(b)

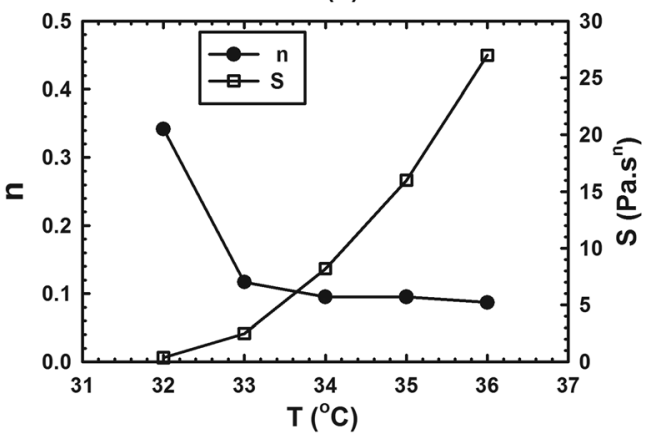

FIG. 5. (a) Storage modulus $\left(\mathrm{G}^{\prime}\right)$ as function of frequency $(\omega)$ at different temperatures for suspensions of $3.61 \mathrm{mg} / \mathrm{ml} f d$-PNIPAM in $I=105 \mathrm{mM}$ buffer (strain amplitude $=0.05$ ). Solid lines are fit to Eq. (4). (b) Slope $n$ and gel strength $S$ obtained from (a) using Eq. (4), as a function of temperature. The solid lines in (b) are to guide the eye. 
followed by slow decrease with temperature shows the importance of the critical gel regime in setting the gel structure.

The effect of temperature on the storage modulus of gels made of stiffer $f d-Y 21 M$ virus particles for a concentration of $4 \mathrm{mg} / \mathrm{ml}$ at $\Delta T=4^{\circ} \mathrm{C}$ is shown in Fig. 6(a). In order to asses the effect of the inherent flexibility of the rods on the rheological properties of the gel, a direct comparison of suspensions of $f d$-virus and stiff $f d-Y 21 M$ at the same concentration of $4.0 \mathrm{mg} / \mathrm{ml}$, both grafted with $10 \mathrm{kDa}$ PNIPAM is made in Fig. 6(b). The particles are identical in terms of their length, diameter, and surface charges, the only difference being the persistence length. On the other hand, the stiffer rods have a lower elastic modulus before gelation, the evolution of the rheological properties during gelation proceeds qualitatively in the same way. The difference between properties of suspensions of the PNIPAM coated $f d$-virus and stiff $f d$ - $Y 21 M$ mutant disappears a $\Delta T=4^{\circ} \mathrm{C}$ above the gel point. Flexibility does not affect the gel strength nor the frequency dependence of these virus particle suspension gels as shown in Fig. 6(b). It can be concluded that at temperatures of $\Delta T=4^{\circ} \mathrm{C}$ and above, the rheological properties of the $f d$-PNIPAM suspensions in the experimentally accessible time and frequency window are fully controlled by the structure of the nascent gel.

The frequency dependence of the moduli of $f d$-PNIPAM gels at $\Delta T=4^{\circ} \mathrm{C}$, hence, is not affected by the flexibility of the building blocks, yet the moduli retain enough of a power-law dependency on frequency, still reflecting the structure of a critical gel. Figure 7 compares $\mathrm{G}^{\prime}$ as a function of frequency for different concentrations of $f d$-PNIPAM at $I=105 \mathrm{mM}$ [Fig. 7(a)] and $1 \mathrm{mM}$ [Fig. 7(b)]. A power law of the moduli with frequency is observed in all experiments. In Fig. 7(a), one experimental data set was extended to frequencies as low as $0.005 \mathrm{rad} / \mathrm{s}$ by using repeated sample loadings and carefully checking for solvent evaporation effects. It can be seen that even when the frequency is lowered to $0.005 \mathrm{rad} / \mathrm{s}$, the slope of $\mathrm{G}^{\prime}$ versus $\omega$ remains constant. The absence of a plateau modulus at low frequencies indicates that the $f d$-PNIPAM gel at $\Delta T=4^{\circ} \mathrm{C}$ is still in the critical gel regime.

The lines in Fig. 7 are fits to Eq. (4). The two resulting fit parameters $S$ and $n$ are listed in Table II for $f d$-PNIPAM gels in buffers with $I=105,5$, and $1 \mathrm{mM}$. As expected, the gel strength $(S)$ increases with particle concentration for a particular ionic strength. The gel strength is highest when the electrostatic interactions are screened most, i.e., for the samples in $I=105 \mathrm{mM}$, consistent with calculations of the interaction potential. Most
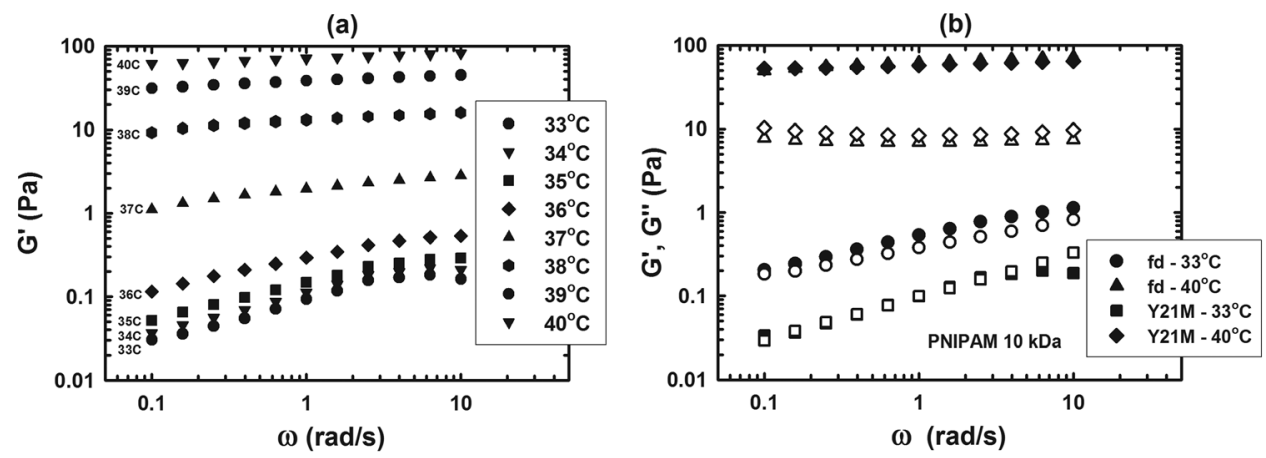

FIG. 6. (a) Storage modulus $\left(G^{\prime}\right)$ as a function frequency $\left(\gamma_{0}=0.05\right)$ at different temperatures for gels composed of stiff $f d-Y 21 M$ virus grafted with $10 \mathrm{kDa}$ PNIPAM in buffer at an ionic strength of $I=105 \mathrm{mM}$. (b) Comparison between the linear viscoelastic storage $\left(\mathrm{G}^{\prime}\right.$, filled symbols) and loss moduli $\left(\mathrm{G}^{\prime \prime}\right.$, open symbols) for semiflexible $f d$-virus $(4 \mathrm{mg} / \mathrm{ml})$ and stiff $f d-Y 21 M(4 \mathrm{mg} / \mathrm{ml})$ grafted with $10 \mathrm{kDa}$ PNIPAM at $\Delta T=-3$ and $4^{\circ} \mathrm{C}$ $\left(\gamma_{0}=0.05\right)$ 
(a)

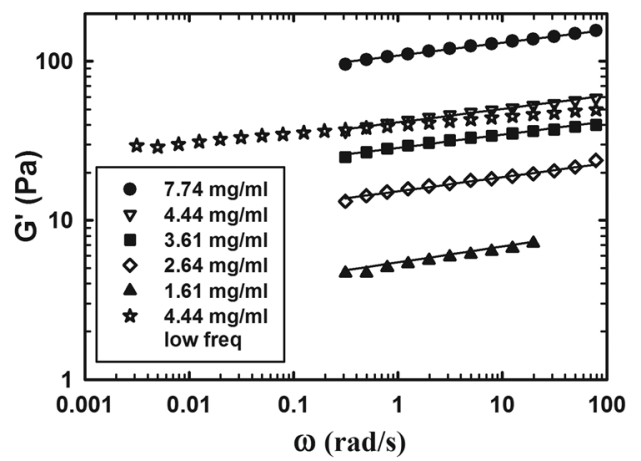

(b)

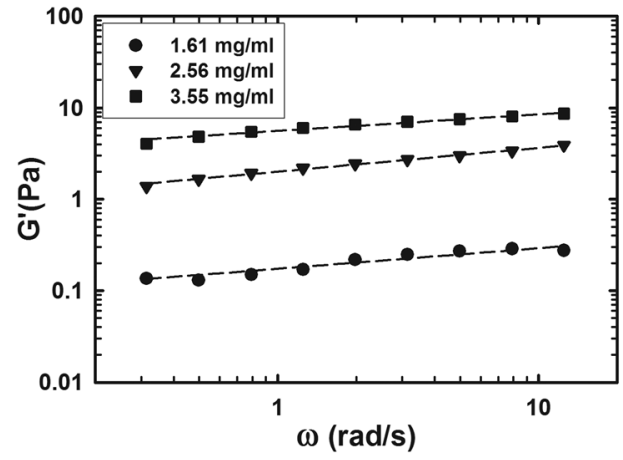

FIG. 7. Storage modulus $\left(\mathrm{G}^{\prime}\right)$ as a function of frequency $(\omega)$ at a strain of 0.05 for different concentrations of $f d$-PNIPAM in buffers at (a) $I=105$ and (b) $I=1 \mathrm{mM}$ buffer. Lines are fits to Eq. (4). One set of data in the low frequency range is shown for $4.44 \mathrm{mg} / \mathrm{ml}$ of $f d$-PNIPAM gel in a buffer with $105 \mathrm{mM}$ ionic strength.

interestingly the values of $n$ for gels at $I=5$ and $I=105 \mathrm{mM}$ differ from those in gels at $I=1 \mathrm{mM}$. The power-law exponent $n$ reflects the nature of the size distribution. A higher value of $n$ means that there is broader aggregate size distribution, consistent with a slightly more "open structure." This can be (qualitatively) attributed to the electrostatic interactions which favor particles approaching each other orthogonally before the particles lock in to form a gel.

\section{Creep}

To extend the strain controlled oscillatory measurements to longer time scales, creep experiments were performed in a constant stress mode. Creep compliance as a function of time are plotted in Fig. 8 for different concentrations of $f d$-PNIPAM in $I=105 \mathrm{mM}$ buffer at $\Delta T=4^{\circ} \mathrm{C}$ for an applied stress of $1.0 \mathrm{~Pa}$. The applied stress of $1.0 \mathrm{~Pa}$ is below the apparent yield stress of the gels. The long time creep compliance data are shown in Fig. 8(a) and the lines are fits to the power-law equation for critical gels given by [Venkataraman and Winter (1990)]

$$
J(t)=\frac{t^{n}}{S \Gamma(1-n) \Gamma(1+n)},
$$

where the parameters $S$ and $n$ have their usual meaning. The values for $S$ and $n$ obtained from the creep tests are listed in Table III. These values are in excellent agreement with those obtained from frequency sweep experiments (Table II).

TABLE II. Strength $S$ and power-law exponent $n$ obtained from frequency sweep experiments using Eq. (4).

\begin{tabular}{|c|c|c|c|c|c|c|c|c|}
\hline $\begin{array}{c}\text { Conc. } \\
(\mathrm{mg} / \mathrm{ml}) \\
105 \mathrm{mM}\end{array}$ & $\begin{array}{c}S \\
( \pm 0.5) \\
105 \mathrm{mM}\end{array}$ & $\begin{array}{c}n \\
( \pm 0.005) \\
105 \mathrm{mM}\end{array}$ & $\begin{array}{c}\text { Conc. } \\
(\mathrm{mg} / \mathrm{ml}) \\
5 \mathrm{mM}\end{array}$ & $\begin{array}{c}S \\
( \pm 0.5) \\
5 \mathrm{mM}\end{array}$ & $\begin{array}{c}n \\
( \pm 0.005) \\
5 \mathrm{mM}\end{array}$ & $\begin{array}{c}\text { Conc. } \\
(\mathrm{mg} / \mathrm{ml}) \\
1 \mathrm{mM}\end{array}$ & $\begin{array}{c}S \\
( \pm 0.5) \\
1 \mathrm{mM}\end{array}$ & $\begin{array}{c}n \\
( \pm 0.005) \\
1 \mathrm{mM}\end{array}$ \\
\hline 1.61 & 6.5 & 0.130 & 2.0 & 5.0 & 0.115 & 1.61 & 0.15 & 0.220 \\
\hline 2.64 & 17.0 & 0.090 & 3.0 & 11.5 & 0.075 & 2.56 & 1.7 & 0.260 \\
\hline 3.61 & 32.0 & 0.080 & 4.1 & 19.0 & 0.095 & 3.55 & 5.0 & 0.180 \\
\hline 4.44 & 46.0 & 0.085 & & & & & & \\
\hline 7.74 & 120.0 & 0.080 & & & & & & \\
\hline
\end{tabular}


(a)

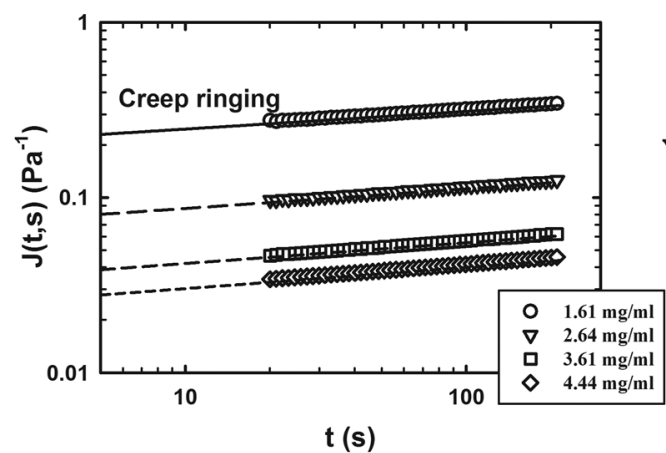

(b)

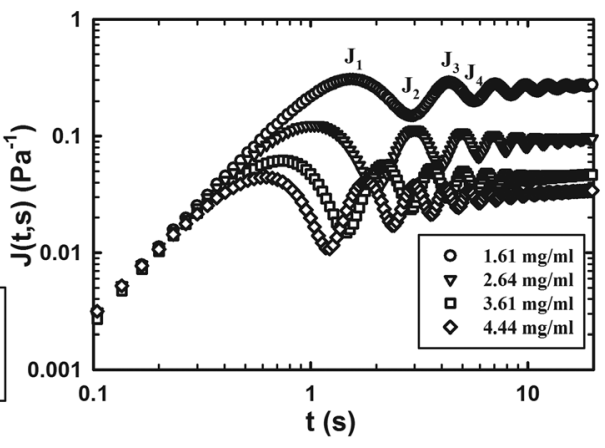

FIG. 8. Creep compliance $(J)$ as a function of time $(t)$ for different concentrations of $f d$-PNIPAM gels in a buffer of $I=105 \mathrm{mM}$ ionic strength at $\Delta T=4^{\circ} \mathrm{C}$ and for an applied stress of $1 \mathrm{~Pa}$. (a) Creep compliance $(J)$ data for times $>20 \mathrm{~s}$, lines are fits to Eq. (5). (b) Creep compliance data for short times where creep ringing is seen.

Figure 8(b) shows creep ringing data obtained at short times. The creep ringing phenomenon occurs due to the coupling between elasticity of the sample and instrumental inertia of the stress controlled rheometer [Ewoldt and McKinley (2007)]. It can be seen from Fig. 8(b) that the ringing frequency and damping of the oscillations increase with the increase in $f d$-PNIPAM concentration. Information about the gel storage and loss moduli can be obtained from the creep ringing data provided the instrument inertia is known [Struik (1967); Zolzer and Eicke (1993); Baravian and Quemada (1998)]. Here a simpler method is used to obtain $G^{\prime}$ and $G^{\prime \prime}$ via Eqs. (6) and (7) following Ewoldt and McKinley (2007),

$$
\begin{gathered}
G^{\prime}=\frac{I \omega^{2}}{b_{c p}}\left[1+\left[\frac{\Delta}{2 \pi}\right]^{2}\right] G^{\prime \prime}=\frac{I_{I} \omega^{2}}{b_{c p}}\left[\frac{\Delta}{\pi}\right], \\
\Delta=2 \ln \left[\frac{J_{1}-2 J_{2}+J_{3}}{-J_{2}+2 J_{3}-J_{4}}\right] b_{c p}=\left[\frac{2 \pi r^{3}}{3 \tan \theta}\right],
\end{gathered}
$$

where $J_{i}$ are the first two peaks and the first two valleys of the creep ringing data, $I_{I}$ is the inertia of the instrument, $\omega$ is the creep ringing frequency, $r$ and $\theta$ are the radius and the cone angle of the measuring device. $G^{\prime}$ and $G^{\prime \prime}$ obtained from creep ringing data at the ringing frequency and from the forced oscillations are listed in Table IV. The two data sets agree well with each other, confirming the robustness of the frequency sweep experiments.

TABLE III. Strength $S$ and power-law exponent $n$ obtained from the fit to the creep compliance data at long times $\left(>20 \mathrm{~s}\right.$ ) for $f d$-PNIPAM gels in $105 \mathrm{mM}$ at $\Delta T=4^{\circ} \mathrm{C}$ and for an applied stress of $1 \mathrm{~Pa}$ using Eq. (5).

\begin{tabular}{lcc}
\hline \hline Conc. $(\mathrm{mg} / \mathrm{ml})$ & $S( \pm 0.5)$ & $n( \pm 0.01)$ \\
\hline 1.61 & 5.0 & 0.10 \\
2.64 & 14.0 & 0.11 \\
3.61 & 30.0 & 0.12 \\
4.44 & 41.0 & 0.12 \\
\hline \hline
\end{tabular}


TABLE IV. Comparison of $G^{\prime}$ and $G^{\prime \prime}$ obtained from creep ringing and forced oscillations at the ringing frequency for different concentrations of $f d$-PNIPAM gels in $I=105 \mathrm{mM}$ ionic strength buffer.

\begin{tabular}{lccccc}
\hline \hline Conc. & $\omega$ & $\begin{array}{c}\text { Creep ringing } \\
G^{\prime}\end{array}$ & $\begin{array}{c}\text { Creep ringing } \\
G^{\prime \prime}\end{array}$ & $\begin{array}{c}\text { Forced oscillation } \\
(\mathrm{Pa})\end{array}$ & $\begin{array}{c}G^{\prime} \\
(\mathrm{Pa})\end{array}$ \\
\hline 1.61 & 2.0 & 6.9 & 0.55 & 5.64 & $\begin{array}{c}\text { Forced oscillation } \\
G^{\prime \prime} \\
(\mathrm{Pa} / \mathrm{ml})\end{array}$ \\
2.64 & 3.2 & 17.0 & 1.05 & 17.12 & 0.60 \\
3.61 & 4.4 & 32.1 & 1.75 & 32.80 & 1.80 \\
4.44 & 5.3 & 46.9 & 2.25 & 49.50 & 3.50 \\
\hline \hline
\end{tabular}

\section{Stress relaxation}

The frequency sweep experiments required long measurement times especially at low frequencies, which resulted in possible interference on the measurements from sample evaporation. The internal consistency of the long time characteristics was confirmed by stress relaxation experiments using a different setup altogether (different solvent trap and geometry). Stress relaxation experiments provide the most direct test of the power-law relation in Eq. (1) [Chambon and Winter (1987)]. Figure 9 shows the stress relaxation as a function of time for a step-strain experiment within the linear viscoelastic region for $3.61 \mathrm{mg} / \mathrm{ml} f d$-PNIPAM gel in a $105 \mathrm{mM}$ ionic strength buffer at $\Delta T=4^{\circ} \mathrm{C}$. The solid line is a power-law fit [Eq. (1)]. The deviation from the power law at long times is due to the lower resolution limit of the torque transducer. The parameters $S$ and $n$ obtained for different concentrations of $f d$-PNIPAM in the linear limit (for strains $\leq 0.1$ ) are listed in Table V. The values for $S$ obtained from stress relaxation experiments are in good agreement with the values obtained from frequency sweep experiments (Table II) and creep experiments (Table III). However, the values of $n$ are slightly larger when compared to those from the frequency sweep experiments.

\section{Summary of linear viscoelastic data}

The analysis of the $f d$-PNIPAM gels in the critical gel regime provided two parameters, the gel strength $S$ and the gel structure $n$. The average values of $S$ and $n$ obtained

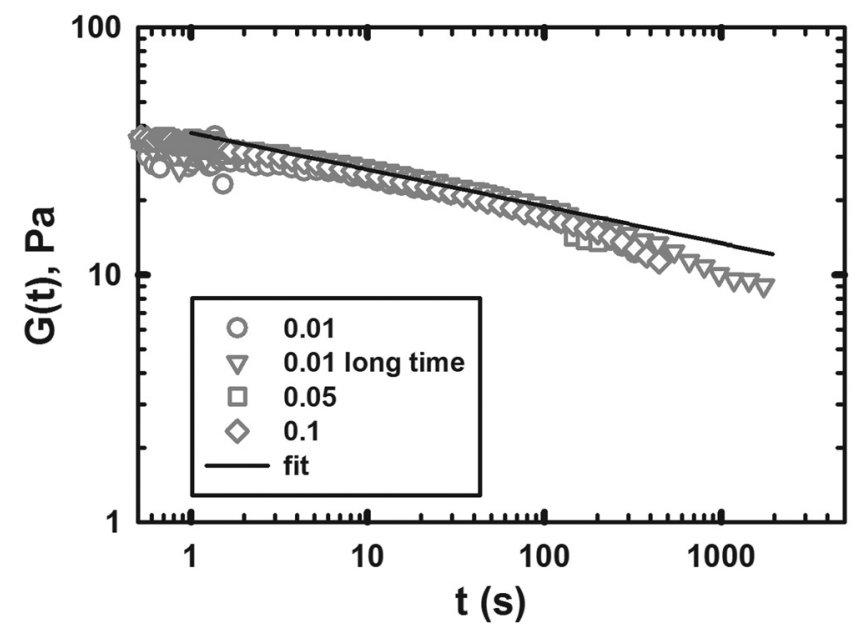

FIG. 9. Stress relaxation modulus $(G)$ as a function of time after step strain with different strain values within the linear limit, for $3.61 \mathrm{mg} / \mathrm{ml}$ of $f d$-PNIPAM gel in $I=105 \mathrm{mM}$ at $\Delta T=4^{\circ} \mathrm{C}$. Full line is a fit to power-law Eq. (1). 
TABLE V. Strength $S$ and power-law exponent $n$ obtained form stress relaxation experiments using Eq. (1) for $f d$-PNIPAM gels in $I=105 \mathrm{mM}$ at $\Delta T=4^{\circ} \mathrm{C}$ and for strains within the linear limit $(<0.1)$.

\begin{tabular}{lcc}
\hline \hline Conc. $(\mathrm{mg} / \mathrm{ml})$ & $S( \pm 0.5)$ & $n( \pm 0.01)$ \\
\hline 1.61 & 7.0 & 0.12 \\
2.64 & 19.0 & 0.15 \\
3.61 & 36.0 & 0.13 \\
\hline \hline
\end{tabular}

from the results of different rheological experiments are plotted in Fig. 10 as a function of concentration of $f d$-PNIPAM particles. The gel strength $S$ increases with increasing concentration due to an increase in the number of contact points or bonds between particles, thus, making the gel stronger. In addition, the gel strength increases from low to high ionic strength buffer as the repulsive barrier is decreased as seen from the interaction potential calculations.

The power-law exponent $n$ that describes the gel structure is plotted as a function of $f d$ PNIPAM concentration in Fig. 10(b). For $f d$-PNIPAM gels at ionic strength of $I=105$ and $5 \mathrm{mM}$ buffer the value of $n \simeq 0.1$ and changes little as the concentration of the particles increases. The $n$ values for $f d$-PNIPAM gels in $1 \mathrm{mM}$ are slightly larger indicating a more open structure. These differences arise because the probability of $f d$-PNIPAM particles to approach each other perpendicularly can be expected to be higher when the ionic strength is lower. Even though the difference in the structure is small between the $f d$-PNIPAM gels, rheological measurements seem to pick up these differences better than scattering.

\section{Linear to nonlinear viscoelastic regime}

In this section, $f d$-PNIPAM gels are characterized during the transition from linear to the nonlinear viscoelastic regime. Two important parameters, i.e., the critical strain amplitude and an apparent yield stress are determined. The critical strain is defined as the strain necessary to break the bonds in the gel and similarly apparent yield stress is defined as the stress beyond which the system behaves as a liquid.

\section{Oscillatory experiments under large strain}

The magnitude of the storage modulus is larger than the loss modulus in strain sweep experiments conducted in the linear regime. As the strain amplitude increases, the gel

(a)

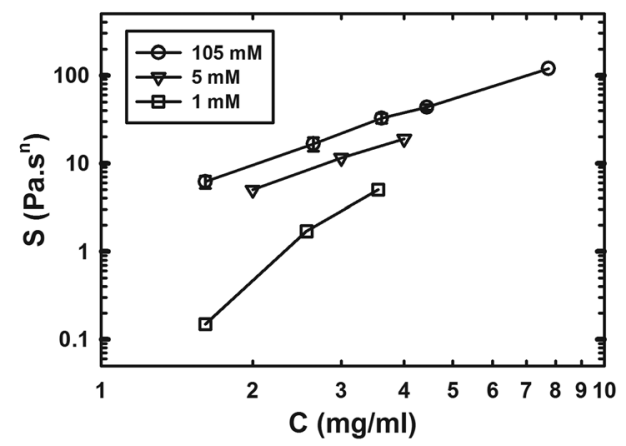

(b)

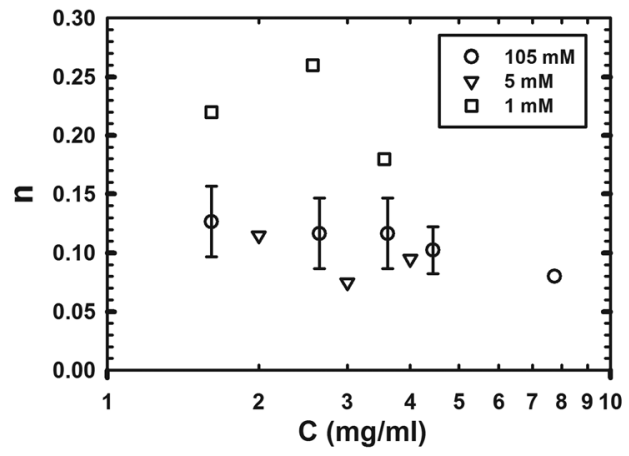

FIG. 10. (a) Strength of the gels $(S)$ as a function of concentration (C) for different ionic strengths. The solid lines are to guide the eye. (b) Structure of the gels $(n)$ as a function of concentration (C). The values of $S$ and $n$ are an average from Tables II-V. 
"yields" to become a liquid, and $G^{\prime \prime}$ becomes larger than $G^{\prime}$ as shown in Fig. 4. The transition from gel to liquid state occurs for strain amplitude larger than a critical strain $\left(\gamma_{c}\right)$. In the nonlinear regime, the physical meaning of the moduli is no longer valid because unlike for small amplitude oscillatory shear, where for a single frequency strain input the stress output will oscillate at the same frequency, for large strain amplitudes the stress output will have higher harmonics [Dealy and Wissbrun (1990)]. The critical strain as a function of $f d$-PNIPAM concentration is shown in Fig. 11, here $\gamma_{c}$ is defined as the strain for which the storage modulus shows a 5\% deviation from the plateau storage modulus. Lines are power-law fits, $\gamma_{c} \propto C^{-b}$. The critical strain is highest for gels in $I=1 \mathrm{mM}$ ionic strength buffer, meaning they are more ductile than the gels in $I=105 \mathrm{mM}$ for which $\gamma_{c}$ is the lowest. Overall, $\gamma_{c}$ decreases as the concentration increases, indicating that stronger gels become more brittle.

\section{Stress relaxation under large step strain}

Stress relaxation experiments in the nonlinear regime are shown in Fig. 12(a) for $3.61 \mathrm{mg} / \mathrm{ml} f d$-PNIPAM gel at an ionic strength of $I=105 \mathrm{mM}$ at $\Delta T=4^{\circ} \mathrm{C}$. In the nonlinear region, stress relaxation modulus $G(t, \gamma)$ is a function of both time and the applied step strain. As can be seen from Fig. 12(a), the $G(t, \gamma)$ data deviate from the linear limit for strains greater than 0.2. A damping function $h\left(\gamma_{o}\right)=\mathrm{G}(\gamma) / \mathrm{G}\left(\gamma_{o}\right)$, shown in Fig. 12(b), is extracted from the relaxation moduli for different concentrations of $f d$-PNIPAM gels as a function of applied step strain. In the construction of the damping function, the stress-strain separability of the stress relaxation data has not been investigated in detail, but it is known to hold for some suspensions [Umeya and Otsubo (1981); Watanabe et al. (1996); Le Meins et al. (2002); Uematsu et al. (2010)]. A sharp downturn in the damping function around a strain of 1 indicates breaking or yielding and is typical for particulate gels [Buscall et al. (1998)]. The strain amplitude required to break the gels determined from the strain sweep experiments (Fig. 11) and from the damping function [Fig. 12(b)] are in good agreement with each other, and both show a decrease in critical strain with an increase in $f d$-PNIPAM concentration, indicating that stronger gels are more brittle.

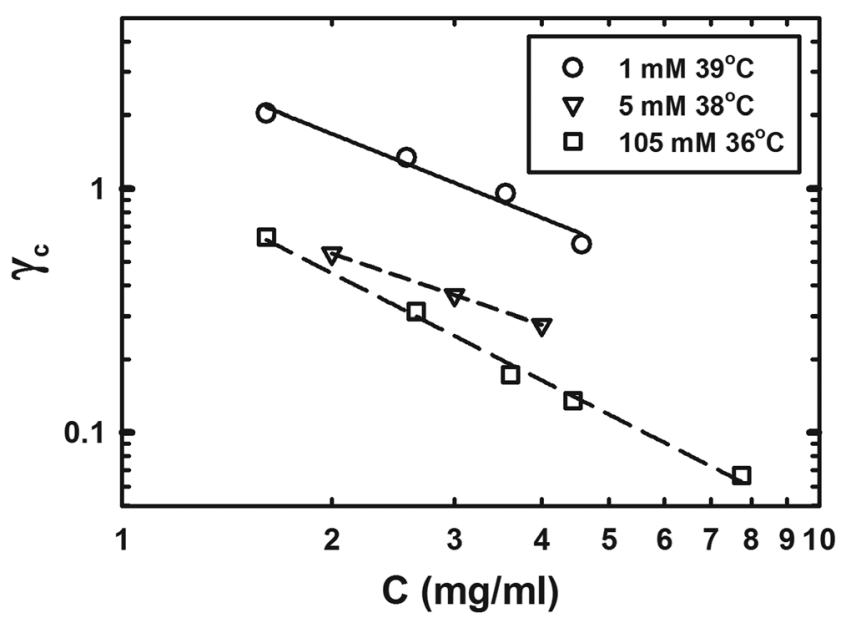

FIG. 11. Critical strains $\left(\gamma_{c}\right)$, obtained from strain sweep experiments performed at a frequency of $6.28 \mathrm{rad} / \mathrm{s}$ are plotted as a function of $f d$-PNIPAM concentration (C) in $I=1,5$, and $105 \mathrm{mM}$ buffers at $\Delta T=4^{\circ} C$. Lines are to guide the eye. 
(a)

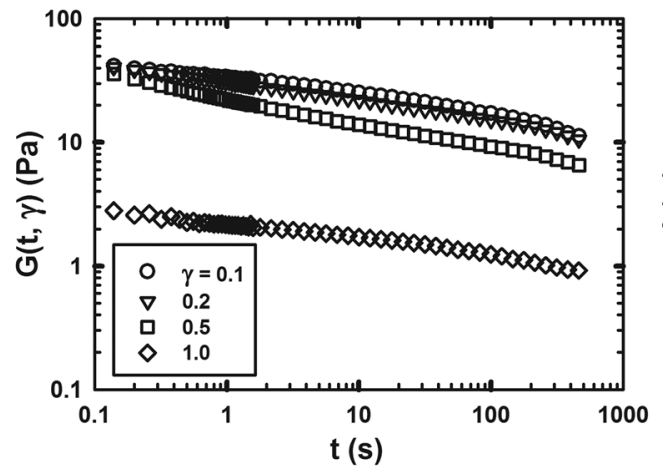

(b)

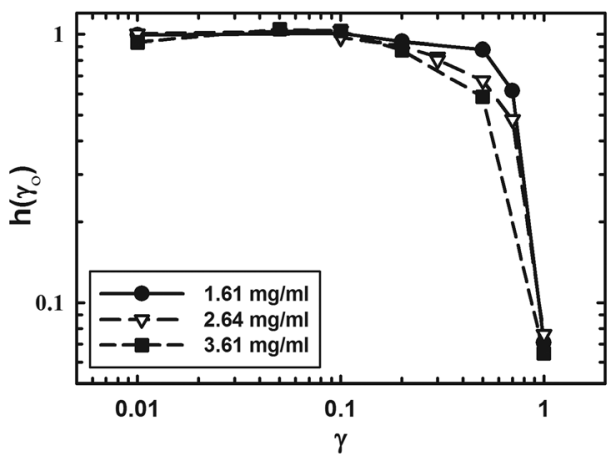

FIG. 12. (a) Stress relaxation $(G)$ as a function of time for different strains for $3.61 \mathrm{mg} / \mathrm{ml} f d$-PNIPAM gel in $105 \mathrm{mM}$ ionic strength buffer at $\Delta T=4^{\circ} \mathrm{C}$. (b) Damping function $\mathrm{h}\left(\gamma_{o}\right)$ as function of applied strain $(\gamma)$ at $1 \mathrm{~s}$ for different concentrations of $f d$-PNIPAM gels in $I=105 \mathrm{mM}$ ionic strength buffer. Lines are to guide the eye.

\section{Apparent yield stress}

Measurements of the apparent yield stress $\left(\sigma_{y}\right)$ experiments were carried out following the method suggested by Möller et al. (2006). The sample was brought to a fixed and reproducible initial condition (by using a temperature history as mentioned in the experimental section) after which a constant stress was applied for a long period of time and the corresponding strain and viscosity were recorded. For an applied stress below the apparent yield stress, the strain in the sample remains small and eventually the flow becomes extremely slow. If the applied stress is slightly higher than the apparent yield stress, the strain in the sample increases rapidly [Coussot et al. 2002]. Figure 13(a) shows the strain as a function of time for different applied stress levels $f d$-PNIPAM suspensions of $4.44 \mathrm{mg} / \mathrm{ml}$ at $I=105 \mathrm{mM}$ at $\Delta T=4^{\circ} \mathrm{C}$. In the experimental time window of $1000 \mathrm{~s}$, an apparent yield stress of $7.5 \pm 0.5 \mathrm{~Pa}$ was obtained [see Fig. 13(a)]. The apparent yield stresses measured in this way for $f d$-PNIPAM gels at various concentrations and ionic strengths are plotted in Fig. 13(b) where the lines are fit to a power-law equation. The apparent yield stress increases with concentration of $f d$-PNIPAM particles for a particular ionic strength and is highest for gels in $105 \mathrm{mM}$ and lowest for gels in $I=1 \mathrm{mM}$, in line

(a)

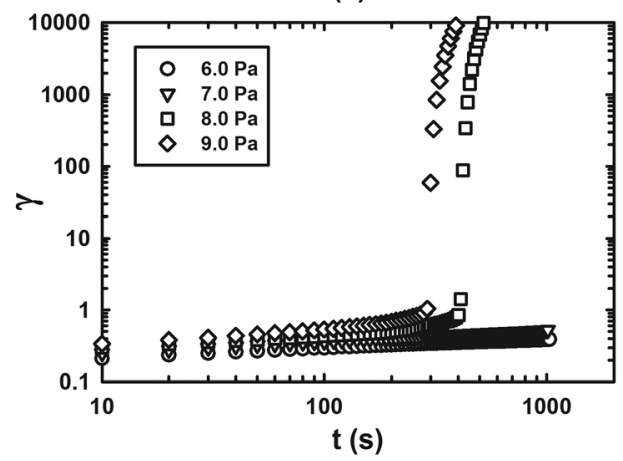

(b)

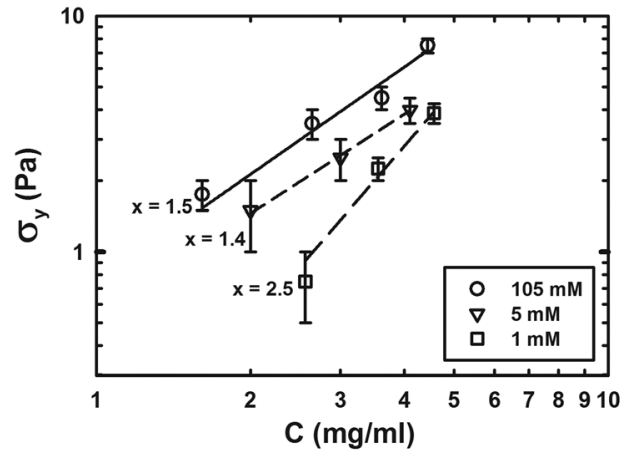

FIG. 13. Strain $(\gamma)$ as a function of time for different applied stress for $4.44 \mathrm{mg} / \mathrm{ml} f d$-PNIPAM gel in $I=105 \mathrm{mM}$ at $\Delta T=4^{\circ} \mathrm{C}$. (b) Apparent yield stress $\left(\sigma_{y}\right)$ as a function of concentration (C) for $f d$-PNIPAM gels in different ionic strengths at $\Delta T=4^{\circ} \mathrm{C}$. Lines are fit to power-law equation and corresponding power-law exponents are shown next to each line. 
with the trends expected from the interaction potential calculations. A yield stress value of $10 \mathrm{~Pa}$ was obtained for gel made of anisotropic rodlike boehmite particles with an aspect ratio of 25 for a volume fraction of 0.0145 [Wierenga et al. (1998)]. Due to the high aspect ratio and different interaction potential of $f d$-PNIPAM particles, the same apparent yield stress value $\left(\sigma_{y}=10 \mathrm{~Pa}\right)$ was achieved at a much lower volume fraction of 0.0048 (three times lower).

\section{Summary of linear to nonlinear transition}

Concluding, characterizing the transition from linear to nonlinear viscoelastic regime for $f d$-PNIPAM gels in the critical gel regime provided two parameters, i.e., the critical strain and apparent yield stress. These parameters describe the necessary amount of strain or stress required to transform the $f d$-PNIPAM gels into a liquid. The critical strains for $f d$-PNIPAM gels in 105 and $5 \mathrm{mM}$ are the lowest at fixed concentration, indicating that these gels are more brittle than the gels at $I=1 \mathrm{mM}$. For $f d$-PNIPAM gels at the same ionic strength, the critical strain decreases with increasing concentration of the particles, due to the increase in the overall brittleness. The stress necessary to transform $f d$-PNIPAM gel into a liquid increases with ionic strength and concentration. The highest apparent yield stress was observed for gels in $105 \mathrm{mM}$ and lowest for gels in $I=1 \mathrm{mM}$.

\section{FINAL REMARKS}

A model thermoreversible colloidal gel made of monodisperse rodlike particles has been studied using rheology and scattering. The attractive interaction causing the gelation is induced by PNIPAM polymer grafted on the surface of the $f d$-viruses. These polymers undergo a hydrophilic-hydrophobic transition for temperatures higher than $\mathrm{T}_{V P T}$. The strength of the gel was tuned by changing the ionic strength of the buffer, thereby controlling the size of the double layer.

Rheological experiments and an analysis for near critical gels were used to assess the strength and structure of the $f d$-PNIPAM colloidal gels at the macroscopic level. In the linear regime, the rheological data were analyzed using critical gel theory that provided two parameters, gel strength $S$ and the gel structure $n$. Rheological characterization of $f d$ PNIPAM gels during the transition from a gel to a liquid state provided the critical strain and apparent yield stress that describes this transition. All measurements show the same trend: gels in a 105 and $5 \mathrm{mM}$ buffer show similar power-law behavior when gel strength $(S)$ and apparent yield stress $\left(\sigma_{y}\right)$ are plotted as a function concentration. When the repulsion between the rods is further increased, there is a jump in the gel strength $S$ and apparent yield stress to a lower value, while at the same time the system becomes less brittle as can be concluded from increase in the critical strain. Moreover, the $n$ value increased by a factor 2 for going between an ionic strength of 105 and 5-1 mM. This larger $n$ for the lowest ionic strength indicates that under these conditions, the $f d$-PNIPAM gels have a slightly more open structure compared to the gels at an ionic strength of 5 and $105 \mathrm{mM}$.

The pronounced increase in the magnitude of the moduli, which is observed between 1 and $5 \mathrm{mM}$ ionic strength, suggests that at $1 \mathrm{mM}$ ionic strength the electrostatic repulsion still contributes significantly, while at $5 \mathrm{mM}$ and higher it is dominated by the PNIPAM attraction. Moreover, there is a remarkable structural resemblance between the gels at 5 and $105 \mathrm{mM}$. These results are surprising, considering the fact that the calculations of effective electrostatic double layer for bare virus particles at ionic strengths of 1,5 , and $105 \mathrm{mM}$ gave effective diameters of $D_{\mathrm{eff}}^{\mathrm{ES}}=63,30$, and $11 \mathrm{~nm}$, respectively, while the collapsed PNIPAM layer is around $2 \mathrm{~nm}$. This observation suggests that the PNIPAM 
layer changes both the local dielectric constant and the "effective" ionic strength close to the particle surface.

It can be noted that for these systems, although the elastic modulus increases with 3 orders of magnitude at $T_{\mathrm{VPT}}$, all experiments at $\Delta T$ up to $4^{\circ} \mathrm{C}$ show that systems are not yet fully developed into solid gels. $\mathrm{G}^{\prime}$ decays in a power-law fashion with decreasing frequency over the entire frequency range, suggesting self-similar structures. Especially for an ionic strength of $1 \mathrm{mM}$, DLS also shows that there is still appreciable internal dynamics, suggesting that the bonding is less strong. If the bonding is Arrhenius like then it can be conjectured that with a strong repulsive force the detachment rate is quite high [Sprakel et al. (2008)]. Mechanically this is reflected in the relatively high critical strain amplitude and low apparent yield stress, both suggesting that there is appreciable internal relaxation possible before the network structure breaks up. Even for an ionic strength of $105 \mathrm{mM}$, where the electrostatic repulsions are completely screened, relaxation processes are still present. This is exemplified by the continuous relaxation in the creep experiments before the system readily flows at around $100 \mathrm{~s}$, see Fig. 13(a). The relaxation times are comparable to the lowest frequency measured in the dynamic test, Fig. 7. Comparing gels made of the wild type $f d$-virus and the stiffer $f d-Y 21 M$ mutants learns that the stiffness of the building blocks ( $f d$-virus and $f d-Y 21 M$ ) does not play a role, and the elastic modulus is set purely by the macroscopic structure of the gel.

Characterization of $f d$-PNIPAM system by scattering revealed a liquidlike structure in the suspension state which was essentially "locked" into a gel state as the temperature was increased. Despite the wide q-range probed, no significant difference could be resolved in the structure as a function of ionic strength. In addition, confocal images show homogeneous density distributions (data not shown). Thus, unlike depletion flocculated systems or in suspensions of attractive microtubules, where bundling is observed [Lin et al. (2007)], no such bundling is seen for our system in all ionic strengths. Instead, the gel networks formed by the sticky $f d$-particles are homogeneous, similar to the crosslinked actin gels [Tharmann et al. (2007)].

To summarize, a model homogeneous and thermoreversible gel made of monodisperse rodlike particles has been studied in the critical gel regime with the aid of rheology and scattering. Gelation of $f d$-PNIPAM system at low ionic strengths $(1$ and $5 \mathrm{mM})$ indirectly showed that the thermoreversible PNIPAM polymer reduces the electrostatic repulsion force significantly. The near critical gel description was able to capture all of the rheological properties in the linear viscoelastic regime. For the gels studied here, rheology proved to be more sensitive to structural changes than a variety of scattering techniques.

\section{ACKNOWLEDGMENTS}

The authors thank Jan Mewis for stimulating discussions. We thank the EU for funding through the project NANODIRECT (Grant No. CP-FP 213948-2). The authors are grateful to European Synchrotron Radiation Facility (ESRF) for providing the beamtime (experiment SC-2201) and Theyencheri Narayanan and Michael Sztucki of ID02 for their assistance with the SAXS experiments.

\section{References}

Alderman, N. J., G. H. Meeten, and J. D. Sherwood, "Vane rheometry of bentonite gels," J. Non-Newtonian Fluid Mech. 39, 291-310 (1991).

Baravian, C., and D. Quemada, "Using instrumental inertia in controlled stress rheometry," Rheol. Acta 37, 223-233 (1998). 
Barry, E., D. Beller, and Z. Dogic, "A model liquid crystalline system based on rod-like viruses with variable chirality and persistence length," Soft Matter 5, 2563-2570 (2009).

Basavaraj, M. G., S. Vandebril, J. Fransaer, and J. Vermant, "Exploiting particle shape in solid stabilized emulsions," Soft Matter 5, 1717-1727 (2009).

Buscall, R., "Letter to the Editor: Wall slip in dispersion rheometry," J. Rheol. 54, 1177-1183 (2010).

Buscall, R., J. I. McGowan, and A. J. Morton-Jones, "The rheology of concentrated dispersions of weakly attracting colloidal particles with and without wall slip," J. Rheol. 37, 621-641 (1993).

Buscall, R., P. D. A. Mills, J. W. Goodwin, and D. W. Lawson, "Scaling behavior of the rheology of aggregate networks formed from colloidal particles,” J. Chem. Soc., Faraday Trans. 84, 4249-4260 (1998).

Chambon, F., and H. H. Winter, "Linear viscoelasticity at the gel point of a crosslinking PDMS with imbalanced stoichiometry,” J. Rheol. 31, 683-697 (1987).

Coussot, P., Q. D. Nguyen, H. T. Huynh, and D. Bonn, “Avalanche behavior in yield stress fluids," Phys. Rev. Lett. 88, 175501 (2002).

Dealy, J. M., and K. F. Wissbrun, Melt Rheology and Its Role in Plastics Processing: Theory and Applications (Van Nostrand Reinhold, New York, 1990).

Dogic, Z., and S. Fraden, “Cholesteric phase in virus suspensions," Langmuir 16, 7820-7824 (2000).

Elson, E. L., "Cellular mechanics as an indicator of cytoskeletal structure and function," Annu. Rev. Biophys. Biophys. Chem. 17, 397-430 (1988).

Ewoldt, R. H., and G. H. McKinley, "Creep-ringing in rheometry: Or how to deal with often-discarded data in step-strain tests,” Rheol. Bull. 76, 4-8 (2007).

Graf, C., H. Kramer, M. Deggelmann, M. Hagenböchle, C. Johner, C. Martin, and R. Weber, "Rheological properties of suspensions of interacting rodlike FD-virus particles," J. Chem. Phys. 98, 4920-4928 (1993).

Grelet, E., and S. Fraden, "What is the origin of chirality in the cholesteric phase of virus suspensions," Phys. Rev. Lett. 90, 198302 (2003).

Hagenböchle, M., B. Weyerich, M. Deggelmann, C. Graf, R. Krause, E. E. Maier, S. F. Schulz, R. Klein, and R. Weber, "Static light scattering by aqueous solutions of rod-like $f d$-virus particles," Phys. A: Stat. Theor. Phys. 169, 29-41 (1990).

Hobbie, E. K., J. A. Fagan, J. Obrzut, and S. D. Hudson, "Microscale polymer-nanotube composites," ACS Appl. Mater. Interface 1, 1561-1566 (2009).

Hoekstra, H., J. Vermant, J. Mewis, and T. Narayanan, "Multi length scale analysis of the microstructure in sticky sphere dispersions during shear flow," Langmuir 21, 11017-11025 (2005).

Huang, F., R. Rotstein, S. Fraden, K. E. Kasza, and N. T. Flynn, "Phase behavior and rheology of attractive rodlike particles," Soft Matter 5, 2766-2771 (2009).

Kheirandish, S., I. Guybaidullin, W. Wohlleben, and N. Willenbacher, "Shear and elongational flow behavior of acrylic thickener solutions. Part I: Effect of intermolecular aggregation,” Rheol. Acta 47, 999-1013 (2008).

Kingery, W. D., H. K. Bowen, and D. R. Uhlmann, Introduction to Ceramics (Wiley-Interscience, New York, 1976).

Lettinga, M. P., Z. Dogic, H. Wang, and J. Vermant, "Flow behavior of colloidal rodlike viruses in the nematic phase," Langmuir 21, 8048-8057 (2005).

Le Meins, J. F., P. Moldenaers, and J. Mewis, "Suspensions in polymer melts. 1. Effect of particle size on the shear flow behavior,” Ind. Eng. Chem. Res. 41, 6297-6304 (2002).

Lin, Y. C., G. H. Koenderink, F. C. MacKintosh, and D. A. Weitz, "Viscoelastic properties of microtubule networks," Macromolecules 40, 7714-7720 (2007).

Matsunaga, T., and M. Shibayama, "Gel point determination of gelatin hydrogels by dynamic light scattering and rheological measurements," Phys. Rev. E 76, 030401(R) (2007).

Maynard, A. D., "Nanotechnology: Assessing the risks," Nanotoday 1, 22-33 (2006).

Mohraz, A., D. B. Moler, R. M. Ziff, and M. J. Solomon, "Effect of monomer geometry on the fractal structure of colloidal rod aggregates," Phys. Rev. Lett. 92, 155503 (2004).

Mohraz, A., and M. J. Solomon, "Gelation and internal dynamics of colloidal rod aggregates," J. Colloid Interface Sci. 300, 155-162 (2006).

Möller, P. C. F., J. Mewis, and D. Bonn, "Yield stress and thixotropy: On the difficulty of measuring yield stresses in practice," Soft Matter 2, 274-283 (2006). 
Ng, T. S. K., and G. H. McKinley, "Power law gels at finite strains: The nonlinear rheology of gluten gels," J. Rheol. 52, 417-449 (2008).

Onsager, L., "The effect of shape on the interaction of colloidal particles," Ann. N.Y. Acad. Sci. 51, 627-659 (1949).

Otten, R. H. J., and P. van der Schoot, "Continuum percolation of polydisperse nanofillers," Phys. Rev. Lett. 103, 225704 (2009).

Panine, P., T. Narayanan, J. Vermant, and J. Mewis, "Structure and rheology during shear-induced crystallization of a latex suspension,” Phys. Rev. E 66, 022401 (2002).

Pelton, R., "Poly( $N$-isopropylacrylamide) (PNIPAM) is never hydrophobic," J. Colloid Interface Sci. 348, 673-674 (2010).

Sambrook, J., and D. W. Russell, Molecular Cloning: A Laboratory Manual, 3rd ed. (Cold Spring Harbor, New York, 2001).

Sato, J., and V. Breedveld, "Evaporation blocker for cone-plate rheometry of volatile samples," Appl. Rheol. 15, 390-397 (2005).

Schild, H. G., "Poly( $N$-isopropylacrylamide): Experiment, theory and application," Prog. Polym. Sci. 17, 163-249 (1992).

Schilling, T., S. Jungblut, and M. A. Miller, "Depletion-induced percolation in networks of nanorods," Phys. Rev. Lett. 98, 108303 (2007).

Schultz, K. M., A. Baldwin, K. L. Kiick, and E. M. Furst, "Gelation of covalently cross-linked PEG-heparin hydrogels," Macromolecules 42, 5310-5316 (2009).

Schulz, S. F., E. E. Maier, M. Hagenböchle, C. Graf, and R. Weber, "Structural properties of dilute aqueous solutions of charged rods studied by light-scattering techniques," Prog. Colloid Polym. Sci. 84, 356-358 (1991).

Solomon, M. J., and P. T. Spicer, "Microstructural regimes of colloidal rod suspensions, gels, and glasses," Soft Matter 19, 1391-1400 (2010).

Sparnaay, M. J., “The interaction between two cylinder shaped colloidal particles," Recl. Trav. Chim. Pays-Bas 78, 680-712 (1959).

Sprakel, J., E. Spruijt, M. A. Cohen Stuart, N. A. M. Besseling, M. P. Letting, and J. van der Guchta, "Shear banding and rheochaos in associative polymer networks," Soft Matter 4, 1696-1705 (2008).

Srivastava, D., C. Wei, and K. Cho, "Nanomechanics of carbon nanotubes and composites," Appl. Mech. Rev. 56, 215-230 (2003).

Stroobants, A., H. N. W. Lekkerkerker, and T. Odijk, "Effect of electrostatic interaction on the liquid crystal phase transition in solutions of rod-like polyelectrolytes," Macromolecules 19, 2232-2238 (1986).

Struik, L. C. E., "Free damped vibrations of linear viscoelastic materials," Rheol. Acta 6, 119-129 (1967).

Tanaka, T., D. Fillmore, S. T. Sun, I. Nishio, G. Swislow, and A. Shah, "Phase transitions in ionic gels," Phys. Rev. Lett. 45, 1636-1639 (1980).

Tang, J. X., and S. Fraden, "Isotropic-cholesteric phase transition in colloidal suspensions of filamentous bacteriophage fd," Liq. Cryst. 19, 459-467 (1995).

Tempel, M., G. Isenberg, and E. Sackmann, "Temperature-induced sol-gel transition and microgel formation in actinin cross-linked actin networks: A rheological study,” Phys. Rev. E 54, 1802-1810 (1996).

ten Brinke, A. J. W., L. Bailey, H. N. W. Lekkerkerker, and G. C. Maitland, "Rheology modification in mixed shape colloidal dispersions. Part I: Pure components," Soft Matter 3, 1145-1162 (2007).

Tharmann, R., M. M. A. E. Claessens, and A. R. Bausch, "Viscoelasticity of isotropically cross-linked actin networks," Phys. Rev. Lett. 98, 088103 (2007).

Uematsu, H., Y. Aoki, M. Sugimoto, and K. Koyama, "Rheology of SiO2/(acrylic polymer/epoxy) suspensions. I. Linear viscoelasticity,” Rheol. Acta 49, 1187-1196 (2010).

Umeya, K., and Y. Otsubo, "Viscoelastic behavior of suspensions of particles in polymer solutions," Polym. Eng. Sci. 21, 619-623 (1981).

van Bruggen, M. P. B., and H. N. W. Lekkerkerker, "Metastability and multistability: Gelation and liquid crystal formation in suspensions of colloidal rods," Langmuir 18, 7141-7145 (2002).

Venkataraman, S. K., and H. H. Winter, "Finite shear strain behavior of a crosslinking polydimethylsiloxane near its gel point," Rheol. Acta 29, 423-432 (1990). 
Verduin, H., B. J. de Gans, and J. K. G. Dhont, "Shear induced structural changes in a gel-forming suspension studied by light scattering and rheology," Langmuir 12, 2947-2955 (1996).

Wachsstock, D. H., W. H. Schwarz, and T. D. Pollard, "Cross-linker dynamics determine the mechanical properties of actin gels,” Biophys. J. 66, 801-809 (1994).

Watanabe, H., M. L. Yao, A. Yamagishi, K. Osaki, T. Shitata, H. Niwa, and Y. Morishima, "Nonlinear rheological behavior of a concentrated spherical silica suspension," Rheol. Acta 35, 433-445 (1996).

Wierenga, A., A. P. Philipse, H. N. W. Lekkerkerker, and D. V. Boger, "Aqueous dispersions of colloidal boehmite: Structure, dynamics, and yield stress of rod gels," Langmuir 14, 55-65 (1998).

Wilkins, G. M. H., P. T. Spicer, and M. J. Solomon, "Colloidal system to explore structural and dynamical transitions in rod networks, gels, and glasses," Langmuir 25, 8951-8959 (2009).

Winter, H. H., "The critical gel: Universal material state between liquid and solid,” NATO Sci. Ser., Ser. C 568, 439-470 (2002).

Winter, H. H., and F. Chambon, “Analysis of linear viscoelasticity of a crosslinking polymer at the gel point," J. Rheol. 30, 367-382 (1986).

Yin, G., and M. J. Solomon, "Soft glassy rheology model applied to stress relaxation of a thermoreversible colloidal gel,” J. Rheol. 52, 785-800 (2008).

Yoshimura, A., and R. K. Prud'homme, "Wall slip corrections for Couette and parallel disk viscometers," J. Rheol. 32, 53-67 (1988).

Zhang, Z., N. Krishna, M. P. Lettinga, J. Vermant, E. Grelet, "Reversible gelation of rod-like viruses grafted with thermoresponsive polymers," Langmuir 25, 2437-2442 (2009).

Zolzer, U., and H. F. Eicke, "Free oscillatory shear measurements-An interesting application of constant stress rheometers in the creep mode," Rheol. Acta 32, 104-107 (1993). 